\title{
Treffsicher
}

\section{Die DRG lanciert zum 01.12.2015 eine Online-Stellenbörse}

Ihr ausgedehntes Netzwerk wird die DRG künftig einsetzen, um Sie auch bei Ihrer Personalsuche wirkungsvoll zu unterstützen. Rund $80 \%$ aller deutschen Radiologen sind über die Fachgesellschaft miteinander verbunden: ein beachtlicher Vorteil für die zielgenaue Suche.

Sie können unter folgenden Angeboten wählen:

\begin{tabular}{|cc|}
\hline Laufzeit & \multicolumn{1}{c}{ Preis } \\
\hline 3 Monate & $300,00 €$ \\
\hline 6 Monate & $400,00 €$ \\
\hline 12 Monate & $650,00 €$ \\
\hline Alle Preise verstehen sich zuzüglich & $19 \%$ MwSt. \\
\hline
\end{tabular}

Zum Einstieg wird in den ersten 3 Monaten ein Rabatt von 50\% gewährt.
Die Anmeldung erfolgt mittels weniger Angaben per Online-Formular. Sie können Ihre Anzeige als pdf-Datei anhängen oder einen Link angeben.

Zugang zur Übersicht der Stellenausschreibungen erhalten Sie direkt über die Startseite der DRG: www.drg.de. Die Angebote sind nach Zeitpunkt der Anmeldung chronologisch geordnet, es ist jedoch eine gezielte Suche nach Fachgebieten (Radiologie, Nuklearmedizin, Strahlentherapie, Strahlenbiologie, -physik und -technik) möglich.

Frau Sabine Strauß (Email: strauss@drg. de, Tel.: 030-916 070 33) steht für Ihre Fragen gerne zur Verfügung. 